\title{
Insuficiencia renal aguda o necrosis tubular aguda en obstetricia
}

\author{
Doctores Saulo Muñoz Delgado (\%) y Rinaldo Mora Restrepo (*) \\ Cali -, Colombia.
}

Han sido muy diversas las denominaciones dadas a esta entidad, las cuales creemos conveniente enumerar para poder identificar exactamente el cuadro clínico. Lucke las agrupó bajo la denominación de "nefrosis de nefrón inferior". Oliver, MacDowell y Tracy la designaron como "falla renal aguda". Bywater y Beall la presentaron como "lesión renal del síndrome de aplastamiento"; otros la llaman "riñón transfusional". Recientemente algunos investigadores propusieron el nombre de "necrosis tubular aguda", designación que nos' parece más apropiada por estar más de acuerdo con la lesión patológica que la caracteriza.

La "necrosis cortical bilateral" es una forma más severa con un cuadro patológico-característico, clínicamente indiferenciable de la "necrosis tubular", cuyo pronóstico es severo y su diagnóstico se hace por medio de estudios anatomopatológicos, ya sea por biopsia o necropsia.

La descripción del cuadro clínico en cuatro períodos definidos, de acuerdo con los recientes conocimientos de la fisiopatología, ha permitido establecer un tratamiento más adecuado para cada uno de éstos y ha mejorado el pronóstico, siendo en la actualidad relativamente benigno.

Trataremos en el presente trabajo de la necrosis tubular aguda como una complicación en obstetricia, ya que en ésta su incidencia es mayor que en cualquier otra especialidad.

En la mayoria de los casos se encuentra el shock como el fac- tor determinante en la producción de la necrosis tubular aguda por la disminución del flujo renal. No importa la causa que la

(*) Auxiliar de Cátedra del Departamento de Obstetricia y Ginecología : de la Fiacultad de Medicina de la Universidad del Valle.

${ }^{* *}$ ) Asistente Residente de la Clínica de Maternidad de Cali. 
produzca, pues tanto en los casos de pérdida masiva de sangre como en la deshidratación severa se produce hipotensión.

En el abruptio placentae además de ser una de las complicaciones más graves tanto para la madre como para el feto, se conjugan factores diversos que producen con gran frecuencia el cuadro de' necrosis tubular aguda. La pérdida masiva de sangre y las sustancias vasopresoras (serotonina) liberadas en el hematoma retroplacentario, así como el shock, los depósitos de la fibrina y el trauma quirúrgico en los casos en que no se presenta el parto, son una suma de factores que hacen más posible la producción de la necrosis tubular aguda.

En el aborto se asocia frecuentemente la hemorragia y la infección, cualquiera de las cuales por sí sola puẹde ser la causa. Además cuando a éste se agrega sepsis por clostridium o streptococus hemoliticus, se presenta la necrosis tubular ya sea por la lesión tóxica directa producida por la toxina o por la hemólisis.

'En la ruptura uterina puede presentarse además del shock y la hemorragia, por la presencia de miohemoglobina liberada por el músculo necrozado que forma cilindros similares a los de la hemoglobina.

En la placenta previa por mecanismo similar al del abruptio placentae concurren además del shock y la hemorragia, el paso de sustancias tromboplásticas debidas al desprendimiento placentario.

En la eclampsia puede presentarse, cuya causa aún no está bien determinada, pero se supone que sea debida a espasmos vasculares. La gran frecuencia de la utilización de sangre total y su inmediata urgencia para tratar muchas de las complicaciones obstétricas hacen frecuente la administración de sangre incompatible o contaminada. La hemolisis que se produce en estos casos causa anoxia, la que según olliver es un mecanismo más importante que la obstrucción mecánica de los túbulos por cristales de hemoglobina.

Dada la relativa frecuencia de la necrosis tubular aguda, consideramos de interés presentar cuatro (4) casos que por su diagnóstico precoz y tratamiento adecuado evolucionaron satisfactoriamente. Al mismo tiempo hacemos una descripción del cuadro clínico y establecemos el tratamiento para cada período de acuerdo con los nuevos conceptos sobre la conveniencia de un balance hídrico adecuado. 


\section{Caso número 1.}

\section{. Casos clínicos}

Historia clínica número 58-11-57.-Clinica de Maternidad̄ de Cali. Nombre: L. M. O. Edad: veinte años. Fecha de ingreso: diciembre $6 / 57$.

Causa de ingreso.-Paciente que consulta por hemorragia vaginal moderada que se inició varios días antes a su ingreso.

Antecedentes obstétricos.-Grávida 1 para 0 . Embarazo a término.

Examen físico-TA: 110/70; pulso: 80 ; temperatura: 37 o.C. Condiciones generales aceptables."

Obstétrico.-Altura uterina: 31 centímetros. Presentación cefálica flotante." Ruidos fetáles normales. Utero de consistencia normal.

En diciembre 12 presentó hemorragia vaginal abundante $\mathrm{y}$ ruidos fetales bradicárdicos, útero hipertónico.

TV. (en condiciones quirúrgicas): Cuello borrado $40 \%$, dilatado 2 centímetros. Se palpa $20 \%$ de reborde placentario desprendido. Presentación cefálica flotante. Membranas integras. Se hace amniotomía y se obtiene líquido amniótico marcadamente meconiado y la presentación no desciende. Se practicó cesárea segmentaria vertical encontrándose placenta previa de un 20\%, desprendida en más de su mitad. Niño vivo de 3.180 gramos.

La evolución del caso se resume en la gráfica número 1.

Patología.Fecha: agosto 12:58. Número M58-25. Nombre: L. M. O. Médico remitente: doctor R. Mora $R$.

\section{Descripción macroscópica:}

El espécimen está constituido por un fragmento de tejido, de forma alargada cil'ndrica (biopsia por punción). Tiene una superficie regular, lisa, de color amarillento. Tiene una longitud de $1 \frac{1}{2}$ centímetros. Se incluye todo el material para su estudio. El material viene conservado en formol (FD):

\section{Descripción microscópica:}

En la biopsia se puede identificar riñón, algunos glomérulos se encuentran aumentados de volumen, llenando casi en su totalidad la cápsula. No hay evidencias de necrosis.

\section{Diagnóstico:}

Riñón derecho, punción biopsia. Ninguno. 710-000. 
488. REVISTA COLOMBIANA DE OBSTETRICIA Y GINECOLOGIA

L.M. $\mathrm{O}$

Historio toi 321459.

CUMICA OE MATEOHOAO Of CALI.

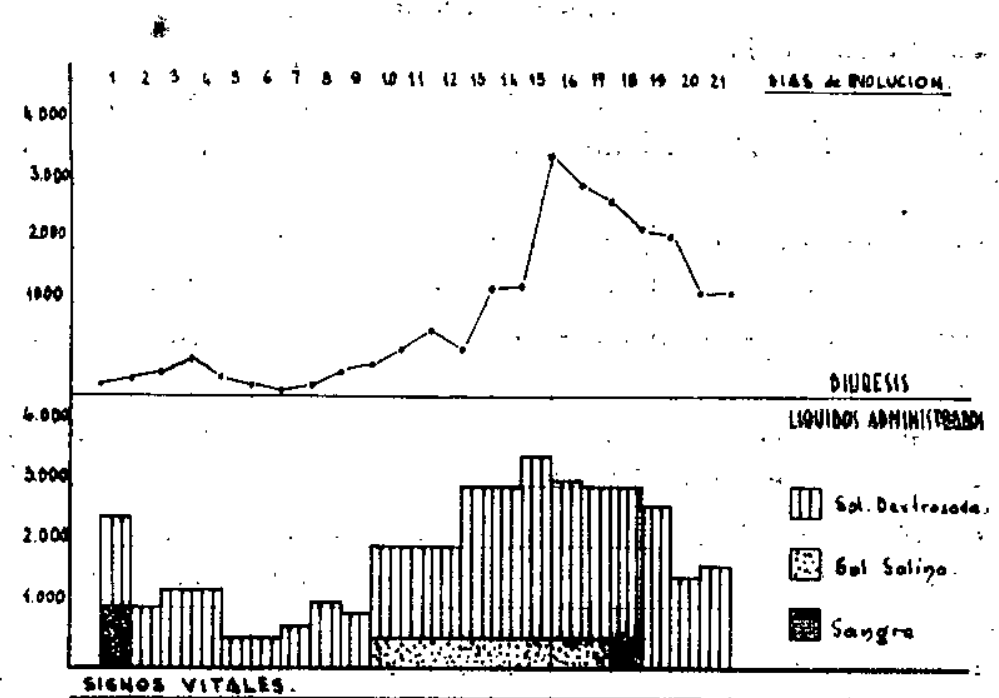

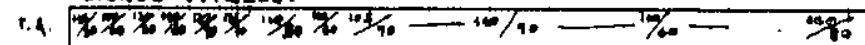

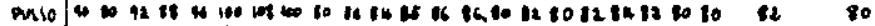

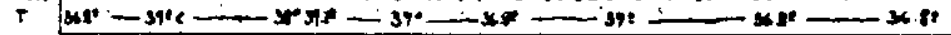

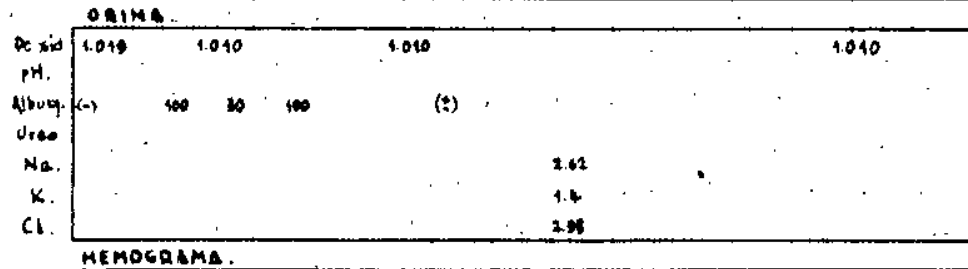

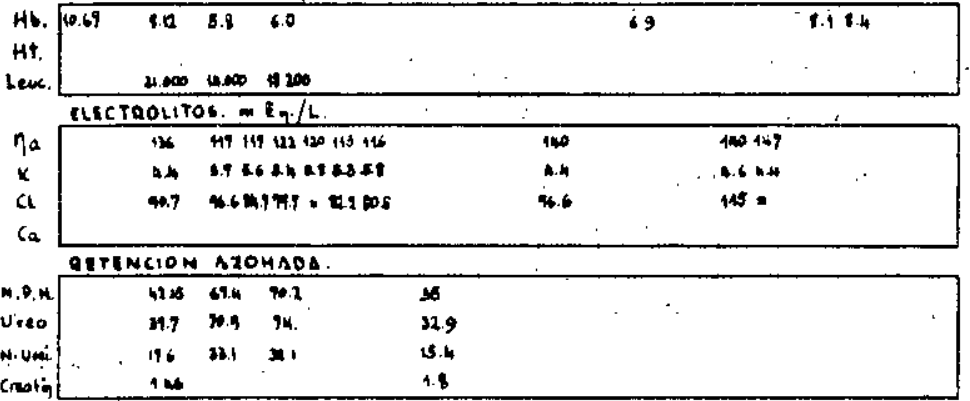

) pquEBAS HEPATICAS.

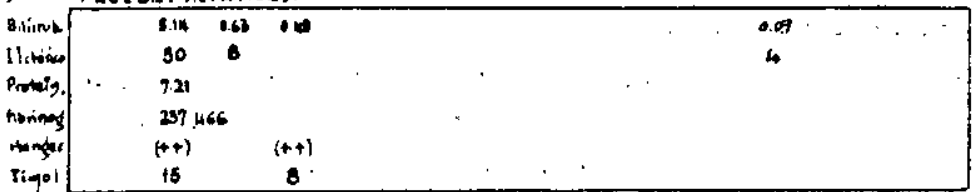

Figura número 1. 


\section{Caso número 2}

Historia clínica número 449-1-58--Clínica de Maternidad de Cali. Nombre: I. V. de H. Edad: veịntitrés años. Fecha de ingreso: enero $24 / 58$. Hora: 7.

Causa de ingreso.-Paciente que consulta por hemorragia vaginal abundante acompañada de dolor hipogástrico, que se inició 20 horas antes del ingreso. Además ausencia de movimientos fetales desde el día anterior a su ingreso.

Antecedentes obstétricos.—Grávida 4 para 1. Embarazo de 26 semanas. Ultima regla: agosto $23-26 / 57$.

Examen físico.-TA: 100/60; pulso: 100; temperatura: $36.6^{\circ}$ C. Paciente en malas condiciones generales, pálida y angustiada.

Obstétrico.-Altura uterina: 24 centímetros, Utero hipertónico, doloroso. Ruidos fetales negativos. No se logran palpar partes fetales por la hipertonía uterina.

TV.-Cuello borrado $40 \%$. Con dilatación de $1,1 / 2$ centimetros. Membranas rotas. Hemorragia moderada. Presentación S I A, completa, alta.

A las 10 horas de su ingreso presentó parto espontáneo con feto de 520 gramos muerto y con un coágulo retroplacentario de 1.060 gramos. La evolución del caso se resume en la gráfica número 2 .

Patología.—Fecha: agosto 12:/58. Número $\mathrm{M}-58-329$. Nombre: I. V. de $H$. Remitente: doctor R. Mora R.

\section{Descripción macroscópica}

El espécimen se encuntra constituído por dos pequeñísimos fragmentos de forma filiforme', de unas 2 décimas de largo, de color blanquecino, consistencia blanda. Son empleados todos para su estudio. Vienen conservados en formol (JA).

\section{Daseripción microscópica}

En las preparaciones se identifica tejido renal que presenta dilatación de los túbulos y cilindros de hemoglobina. Los glomérulos son normales.

Diagnóstico:

Riñón, biopsia. Falla renal aguda. (Nefrosis de nefrón inferior) (Nefrosis hipóxica). 713-38 x 9 . 


\section{IV \\ C. de Madernidaa}

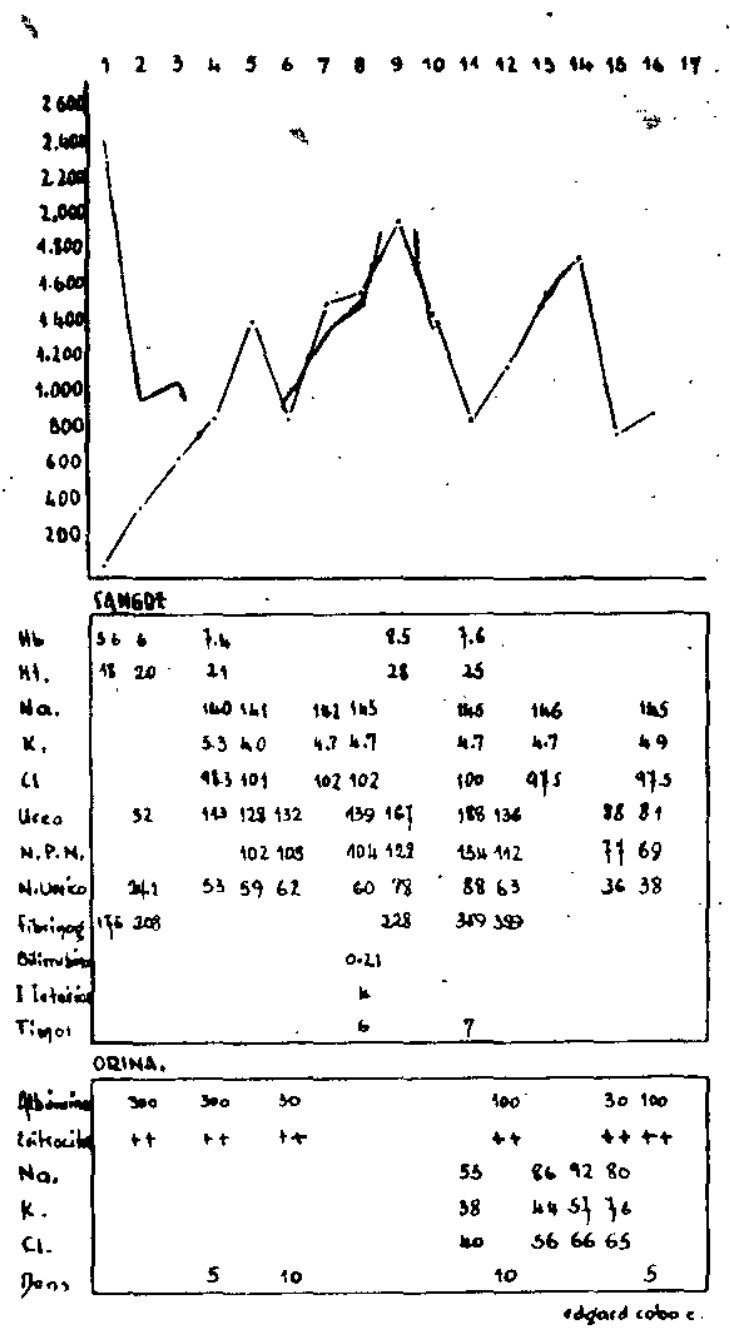

F'igura número 2

\section{Caso número 3}

Historia cl nica número 171,-614 I. C. S. S. Nombre: C. F. Edad: treinta y tres años. Fecha de ingreso: febrero $26 / 58$.

Paciente a quien en febrero 26/58 se li practicó salpingectomía derecha, resécción cuneiforme de ambos ovarios y miomecto- 
C. $f$

kss.
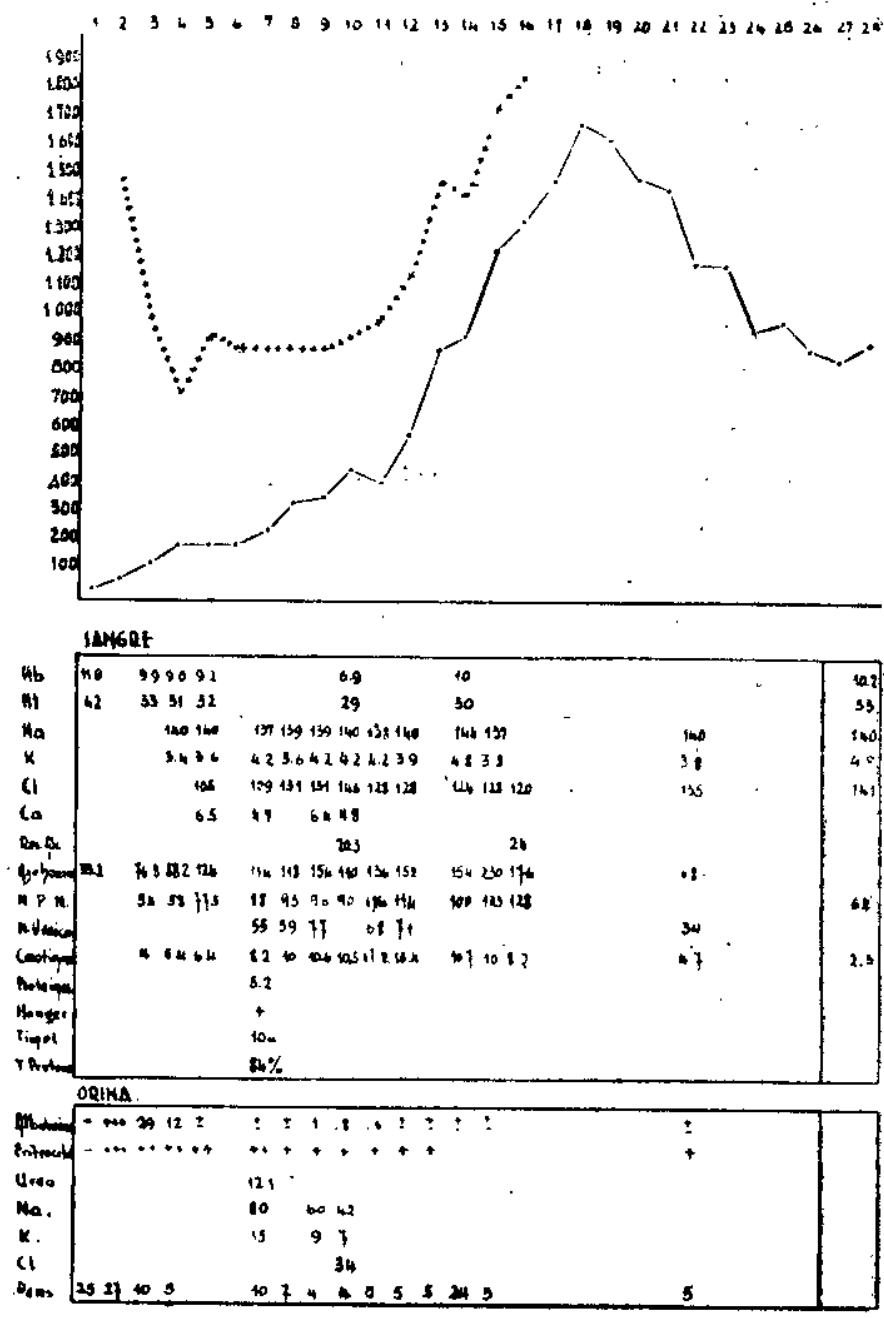

Figura ıúmerọ 3

adjord cabe $c$ :

mía debido a hemotosalpinx derecho, quistes foliculares bilaterales y pequeño mioma subseroso. Durante el acto quirúrgico se la administraron 250 c. cc. de sangre incompatible.

La evolución se resume en la gráfica número 3 . 


\section{Caso número 4}

Historia clínica número 363-6-58.-Clinica de Maternidad de Cali. Nombre: O. G. Edad: cuarenta y seis años. Fecha de ingreso: junio 19/58. Hora: 11 .

Causa de ingreso.-Eclampsia con 3 ataques convulsivos que se habia iniciado el día anterior a su ingreso. Además hemorragia vaginal abundante.

Antecedentes obstétricos.-Grávida 9 para 5. Embarazo de 38 semanas, con feto vivo.

Examen físico.-TA: 250/120; pulso: 90; temperatura: 37.29 C. Edemas: ++++ . Albuminuria: ++++ . Paciente comatosa.

TV.-(En condiciones quirúrgicas y con doble equipo). Cuello borrado $70 \%$, con dilatación de $1 \frac{1}{2}$ centímetros. Placenta previa $100 \%$ (central total). Presentación S. I. A. incompleta. Al día siguiente a su ingreso se practicó cesárea segmentaria longitudinal con anestesia local, debido a hemorragia vaginal abundante. Se obtuvo niño vivo de sexo masculino de 2:620 gramos. En las primeras 24 horas que siguieron a la intervención, sólo eliminó 100 c. c. de orina hemoglobinúrica.

La evolución del caso se resame en la gráfica número 4.

Patología.-Fecha: agosto 12/58. № M58-2123: Nombre: O. G. Médico remitcnte: doctor $R$. Mora $R$.

\section{Descripción macroseópica}

El espécimen está constituido de un pequeño fragmento de tejido de forma cilíndrica. Mide 0.5 de longitud, de color amarillo y consistencia blanda. Se envía todo para iestudio (AR).

\section{Descripción microscópica}

En la preparación estudiada se identifica tejido renal. En los glomérulos se aprecia una fina reticulación. Los túbulos muestran vacuolización en la luz, presencia de albúmina. En el epitelio tubular se advierte aumento de los núcleos, la cual expresa un sigro de regeneración: Las arteriolas muestran sus paredes muy engrosadas:

El cuadro clínico está caracterizado por cuatro per odos bien identificados aun çuando la duración de cada uno. es variable. 


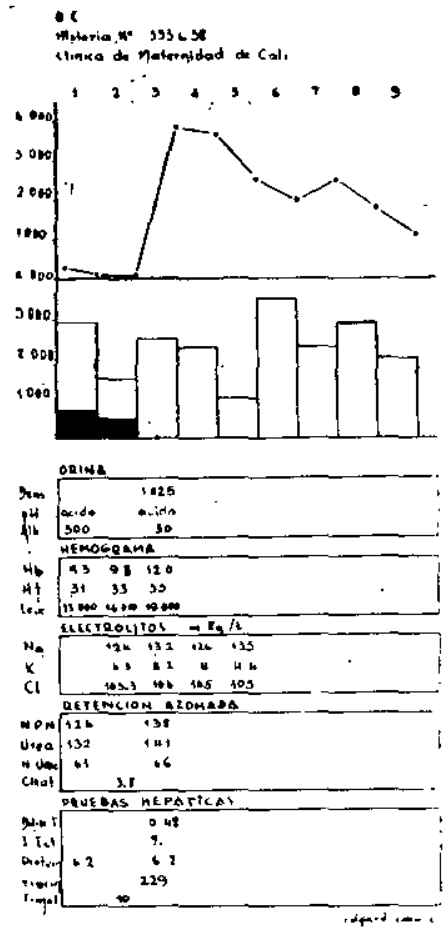

Figura número 4

\section{Cuadro clínico}

1er. período: inicial o de traumatismo.-Anteriormente se mencionaron las causas más frecuentes que desencadenan el cuaaro de necrosis tubular aguda. Este periodo generalmente se caracteriza por una fase de shock. Durante est periodo es dificil establecer si la anuria u oliguria son debidas a la disminución del flujo renal y sólo cuando se ha restablecido la tensión arterial se puede asegurar si se ha instalado un cuadro die lesión renal. Sin embargo en algunos pacientes se puede presentar este periodo inicial sin hipotensión, como en los casos de abruptio placentae o. sepsis severa.

\section{Período inicial}

\begin{tabular}{ll} 
Nombre. & Traumatismo \\
L. M. O. & Placenta previa \\
I. V: de H: & Abruptio placentae \\
IC. F. & $\ldots$ Incompatibilidad sanguinèa \\
O. G. & Incompatibilidad sanguínea \\
\hline
\end{tabular}


20 Periodo: anuria u oliguria. - Él término anuria denota una teliminación urinaria inferior a. 100 c. c. en:24 horas y oliguria una eliminación menor de 350 a 400 c. c. Su duräción es variable y se han reportado casos con períodos de lia 21 días. Durante este tiem-. po hay disminución del flujo renal $y_{;}^{\prime}$ de la filtración glomerular $y$ se establece la necrosis tubular.

Este periodo se considera hasta que: la eliminación de orina alcanza 1:000 c. c. en las 24 horas. En este periodo generalmente las determinaciones de sodio sérico muesträn niveles bajos, no pọr pérdida de este ión sino por paso al compartimento extravascular para reemplazar al potasio celulaz. El'nivel sérico de potasio asciende en ocasiones hasta niveles de hiperpotasemia ( + de 5.5 mEq., por el uamento del metabolismo proteico.

El nivel sérico de calcio se reduce por aumento de los fosfatos y cuando se corrigie en forma rápida la acidosis, por disminución del calcio ionizado. En algunas ocasiones la hipocalcemia es tan marcada que puede llevar a la tetania. La acidosis casi siempre acompaña al período de anuria y es debida a la acumulación de sulfatos, fosfatos y ácidos orgánicos nesultantes de la destrucción celular.

Los productos nitrogenados se acumulan debido a la baja de-puración nenal de éstos y la úrea sérica puede continuar elevándose aún hasta en los primeros días del período de diuresis.

Algunos autores le dan importancia a la relación

concentración úrea sérica

concentración úrea urinaria

que normalmente es de 75/1. Cuando esta relación es inferior a 10 el pronóstico es muy grave y es frecuente encontrar esta relación inferior en este periodo en los casos severos.

Debido a la inactividad de los túbulos, la densidad de la orina permanece en el nivel de isostenuria, ya que el filtrado glomerular es solo ligeramente alterado a través de éstos. A pesar del escaso-volumen urinario la densidad es baja. 


\section{Período de oliguria o anuria}

\begin{tabular}{|c|c|c|c|c|c|c|}
\hline \multirow{2}{*}{ Hombre } & \multirow{2}{*}{$\begin{array}{l}\text { Oliguria } \\
\text { días }\end{array}$} & \multicolumn{2}{|c|}{$\begin{array}{l}\text { Retención nitrogenada Mgr. } \% \text {. } \\
\text { días y niveles más altos }\end{array}$} & \multicolumn{3}{|c|}{$\begin{array}{c}\text { Electrolitos } \mathrm{mBq} / \mathrm{L} \text {. días o niveles } \\
\text { más altos y bajos }\end{array}$} \\
\hline & & N.'P. N. & Greatinina & Sodio & Potasio & Cloro \\
\hline \multirow[t]{2}{*}{ L. M. O. .. . } & 10 & $7-70.2$ & $12-1.8$ & $5-117$ & $5-5.9$ & $10-80.5$ \\
\hline & & & & $23-147$ & $23-4.4$ & $22-115$ \\
\hline \multirow[t]{2}{*}{ I. V. de H... } & 2 & $11-154$ & & $1-140$ & $4-5.3$ & $7-102$ \\
\hline & & & & $13-146$ & $7-4.7$ & $13-97.5$ \\
\hline \multirow[t]{2}{*}{ C. F..$\quad \therefore$} & 9 & $15-133$ & $11-17.2$ & $7-137$ & $4-3.4$ & $5-105$ \\
\hline & & & & $14-144$ & $14-4.8$ & $10-146$ \\
\hline \multirow[t]{2}{*}{ o. G. .." } & 3 & $4-138$ & $4-3.8$ & $4-124$ & $5-4.4$ & $6-105$ \\
\hline & & & & $5-135$ & $4-4$ & $3-103$ \\
\hline
\end{tabular}

CUADRO NUMERO 2

$3^{0}$ período: Poliuria o Diuresis.-Se inicia cuando la eliminación urinaria alcanza $1.000 \mathrm{c}$. c. Durante este periodo el flujo sanguíneo renal y la filtración glomerular se han restablecido, pero aún continúa alterada la función tubular. La eliminación urinaria es de 3: a 5 litros o aún más en las 24 horas, cuya composición es ligeramente diferente a la del filtrado glomerular, a lo cual la úrea sérica continua elevándose aun varios días después de establecida la poliuria, por 10 que no es recomendable dar alimentos con contenido nitrogenado.

En este período se produce gran pérdida de iones y líquidos que hace necesario controles cuidadosos de electrolitos diarios para evitar la hipopotasemía y la deshidratación.

4\% período: Regenerativo o de recuperación.-Pasada la fase de poliuria la eliminación urinaria empieza a decrecer a niveles normales, debido a que se está verificando la regêneración del epitelio tubular, la que puede durar tanto como 200 días, para el restablecimiento completo de la función tubular.

\section{Patología}

Aun cuando son muy diversas las denominacione's dadas a esta entidad, sinembargo la mayoria de los autores concuerdan en la descripción de los hallazgos patológicos. Como todos nuestros casos sobrevivieron, nuestra información se limita a la presentación 
die las biopsias hepáticas y renales obtenidas por punción con la aguja de Vin-Silverman, de acuerdo con las técnicas que describimos en trabajos anteriores.

El cuadro renal microscópico que encontramos es similar a los ya descritos por autores conocidos.

No discutiremos la utilidad y los peligros de la punción-biopsia, ya que creemos que en un plan serio de investigación se pueden obtener sin grandels riesgos para la paciente. En los casos que presentamos no hubo complicación alguna a pesar de sus condiciones y de que las verificamos casi simultáneamente con las biopsias hepáticas.

Practicamos las biopsias hepáticas para tratar de comprobar ios hallazgos descritos por Doniach y Walker en 1946, quienes iencontraron asociadas a la necrosis tubular aguda, lesiones de necrosis en hipófisis, hígado y páncreas.

En uno de los casos (M-58-25) en que encoritramos lesiones patológicas características de necrosis tubular aguda la biopsia hepática no mostró alteración histológica. Én la otra biopsia hepática tampoco encontramos alteración alguna. El cuadro histologico es similar en todos los casos, no importa el factor etiológico. Se caracteriza por degeneración o necrosis de las células del epitelio tubular, lesión que es más severa en el túbulo contorneado proximal. Los túbulos muestran un grado variable de dilatación $\mathrm{y}$ el lumen tubular contiene pigmento granular (cilindros de hemoglobina). Eil hallazgo de estos cilindros no justifica el diagnóstico de necrosis tubular aguda, ya que también se encuientran asociados a enfermedades infecciosas y no son responsables de una mayor alteración de la función renal. El tejido intersticial muestra edema principalmente en la corteza. También se observan focos de células inflamatorias (células plasmáticas, linfocitos e histocitos) en las médula y unión cortico-medular, aunque también puleden invadir la corteza.

Prop

Biopsia renal.- Hallazgos anatomo-patológicos

Nombre: L. M. O.

Algunos glomérulos se encuentran aumentados de volumen y llenan casi en su totalidad la cápsula. No hay evidencia dè necrosis.

Nombre: L. V. de.H. 
Los túbulos aparecen dilatados y contienen cilindros de hemoglobina. Los :glomérulos son normales.

Nombre: $\mathrm{O} . \mathrm{C}$.

En los glomérulos se aprecia una fina reticulación. Los túbulos muestran vacuolización de la luz y presencia de albúmina. En el epitelio tubular se advierte aumento de los núcleos, lo cual expresa un signo de regeneración. Las arteriolas muestran sus paredes muy engrosadas.

\section{Traţamiento.}

El tratamiento de la necrosis tubular aguda es difícil y complejo debido a que cada período requiere cuidado diferente, por lo cual para mayor comprensión consideramos cada fase por separado.

ler. periodo inicial o traumático.-El tratamiento debe encaminarse especialmente a la prevención de la necrosis tibular aguda y tener un conocimiento bastante exacto de su manejo. En caso de que exista hipotensión debe tratarse ésta con la administración de sangre u otra clase de líquidos cuando estén indicados. Así mišmo la infección debe ser combatida. En caso de Abruptio Placentae debe evacuarse el útero a la mayor brevedad antes de que se instalen las lesiones renales.

Cuando ocurre reacción hemolítica transfusional se debe suspender inmediatamente la sangre, extraer sangre de otra vena para realizar la prueba cruzada y buscar hemolisis. So debe forzar la diuresis para evitar el taponamiento de los túbulos por cristales de hemoglobina y para tal iefecto administrar solución dextrosada al 5-10\% en cantidad de 2.000 c. c. y alcalinizantes (solución bicarbonatada al 5\%, lactado de' sodio 1:/6 M.). Esta medida es de beneficio sólo si se aplica muy precozmente. Otros recomiendan la administración de 50 a 100 c. c. de manitol al $25 \%$ que produce diuresis osmótica. También es cle beneficio sólo si se administra inmediatamente. Se han utilizado otras medidas para restaurar el flujo urinario, tales como la denervación y decapsulación renales, la anestesia espinal, la diatermia sobre la región. lumbar, la administración de procaina en una vena periférica o en el pedículo renal, pero sus resultados benéficos aún no han sido plenamente demostrados.

$2^{0}$ período: de anuria u oliguria. Líquidos-Nuevas orientaciones en el tratamiento de la necrosis tubular aguda nos han demos- 
trado plenamente el papel benéfico que desempeña la restricción de liquidos en el pronóstico de esta entidad. J. C. Bacala y B. $\boldsymbol{C}$. Portuondo en 1955 presentaron su informe con 2 series de casos tratados de diferente manera. En una de las series administraron abundante cantidad de líquidos y ninguno de los pacientes sobrevivió; en la otra serie administraron solamente de 500 a 600 c. c. para reemplazar las pérdidas insensibleš. En nuestros casos de acuerdo con las indicaciones del doctor H. J. Tatum, permi-timos 0.3 c. c. hora-kilo-día para reemplazar pérdidas insensiblez, administrados en forma de solución dextrosada por via oral. El objeto de esta miedida es mantener a los pacientes preferiblemente en un bajo nivel de hidratación que sobrehidratarlos, lo que podría conducir al edema pulmonar agudo. En presencia de vómito se puiede administrar solución dextrosada al $15 \%$ por vía endovenosa.

Dieta-Como ya se estableció atrás, se reduce únicamente a la administración de carbohidratos durante los dos primeros periodos.

Electrolitos. - Sodio en anuria no se debe administrar porque ro se excreta. Potasio en cualquier condición en la que la eliminación urinaria sea muy pequeña existe el peligro de la hiperpotasemia.

Se utilizan varias medida's para tratar la hiperpotasemia, de las cuales las más aconsejables son:

1. La administración de solución dextrosada con o sin jnsulina.

2. La absorción y luego eliminación del potasio en el trayecto gastro-intestinal con resinas de cambio catiónico, en dosis de 20 a 60 gramos al día.

3. La dialisis peritoneal.

4. El uso del riñón artificial.

Ratención Azohada.-Para trátar de disminuir la retención azohada se debe administrar una dieta exclusivamente a base de carbohidratos. En el caso de que làs cifras alcancen niveles altos $y$ en que el coma sea inminente, mientras se logra la regeneración de los túbulos se pueden utilizar los procedimientos de vividialisis, tales como lavados gástricos, dialisis pertoneal o el riñón artificial. 
Antibióticos - Se deben adiministrar cuando están indicados, evitando el uso de oxitetraciclina y estreptomicina debido a su efecto acumulativo.

Sangre.-En caso de que la anemia produzca complicaciones y ponga en peligro la vida de la paciente, se puede administrar sangre preferiblemente en forma de concentrado globular. La sangre total contiene alrededor de 170 gramos de proteína por litro que al metabolizarse producirá aumento del nitrógeno y del potasio, razón por la cual sólo debe utilizarse en casos rigurosamente necezarios.

Testosterona.-Algunos recomiendan administrar testosterona en los primeros días de anuria en dosis de 200-40 gramos al día: La testosterona activa la regeneración tubular y retiene potasio en las células evitando 'el paso de éste al compartimiento intravascular.

$3^{\circ}$ período: Diuresis o Poliuria.-En esta fase más daño se causa por deficiencia en el tratamiento que por exceso en la administración de líquidos. Las principales complicaciones son la deshidratación, la hipopotasemia y la hiponatremia. Se deben reemplazar los l'quidos eliminados así como el sodio y el potasio perdidos.

\section{Comentarios}

La necrosis tubular aguda es una entidad que se presenta con relativa frecuencia en la práctica obstétrica. Sus principales factores etiológicos son Abruptio Placentae, placenta previa, aborto séptico, hemorragia, eclampsia, transfusión incompatible, complicación quirúrgica y otras de menor frecuencia.

El cuadro clínico está caracterizado por 4 períodos perfectamente definidos: periodo inicial o traumático, anuria, diuresis o poliuria y recuperación final. El pronóstico es benignoi an la mayoria de los casos siempre y cuando el tratamiento sea adecuado. La base del tratamiento es reemplazar únicamente las pérdidas insensiblés calcüladas en 500 c. c: más las sensibleś," hasta que se inicie la fase de poliuria y de recuperación.

El cuadro anatomo-patológico se' caracteriza por lesiones tubulares. .

Nota.-Los controles de ielectrolitos fueron practicado Laboratorio de Investigación del Departamento de Obstetricia y 
Ginecologia de-la Universidad del Valle, bajo la dirección del doctor H. J. Tatum. Los diemás exámenes fueron practicados en el Laboratorio: de la Clinica de Maternidad.

De los casos clínicos presentados tres se atendieron en la Clinica de Maternidad y el otro en el Departamento de Obstetricia deI I. C. S. S. de Cali, en donde contamos con la valiosa colaboración del doctor Edgar Cobo.

\section{BIBLIOGRAFIA}

Abboterapia, reimpreso número 151,1955 . Lo placenta humana.

ALLEN, A.-Enfermedades del riñón. Editoriul Interamericana, S. A. México, páginas 246, 262.1952.

J. C. BACALA y PORTUONDO B.-Obstetrics and Gynecology. 5: 283. 1955.

Color Atlas of Pathology.--J. B. Lippincott Company.

CUADROS A. y MORA R.-Biopsia renal con Toxemia dol embarazo. Revista Colombiana de Obstetricia y Ginecologia: IX: 143. 1958.

CHAMBERS J. W., and SMITH G.-The British Journal of Surgery. XLV. 160: 1957.

CHESLEY LEON and MCCAW W.-American Journal of Obstetrics and Gynecology 62: 1187. 1951.

GRU I., MUÑOZ S. y MORA R.-Biopsigs del hígado y pruebas de función hepática en toxemias del emparazo. Revista Colombiana de Obstetricia y Ginecologia. IX: 149. 1958.

HERBUT, P.-Pathology. Lea and Febiger Philadelphia, P. 814, 816. 1955.

HOLAND E. y BOURNE A.-British Obstetrics and Gynecological Practice. William Heinemann...Medical Books Ltd. Londres. 264. 1955.

KOEFF W. J.--The Medical Clinics of North America, 39: 1041. 1955.

LAULER D. P. y SCHREINER G. E.-The American Journal of Medicine, XXIV: 159. 1958.

McHINGLY.-The Lancet I: 1957 (mayo 1958)..

MŨNOZ D. S.-Complicaciones de las Toxemias del Embarazo. Abruptio Placentae.. Revista Colombiana de Obstetricia y Gincología. IX: 201. 1958.

PERLMUTTER M.-Annals J. Internal Medicine. 47: 81, 1957:

PRIDDLE H. and STEVENSON C.-Am J. of Obst. and Gynecol. 62: 1053. 1951.

TATUM, H. J. y CUADROS A.-Insuficiencici renal aguda. Nuevos conceptos terapéuticos. Publicación mimeografiada. del Departamento de Obstetricia y Ginecología de la Facultad. de Medicina, Universidad del Valle: 1958. 